\title{
Characterization of aggregate load and pattern in living yeast cells by flow cytometry
}

Itahisa Hernández Hidalgo ${ }^{1, *}$, Thomas Fleming ${ }^{1,2, *}$, Volker Eckstein ${ }^{3}$, Stephan Herzig ${ }^{4,5}$, Peter P. Nawroth ${ }^{1,2,5}$, and Jens Tyedmers ${ }^{1}$

${ }^{1}$ Department of Medicine I and Clinical Chemistry, University of Heidelberg, Heidelberg, Germany, ${ }^{2}$ German Center for Diabetes Research (DZD), München-Neuherberg, Germany, ${ }^{3}$ Department of Medicine V (Haematology), University of Heidelberg, Heidelberg, Germany, ${ }^{4}$ Institute for Diabetes

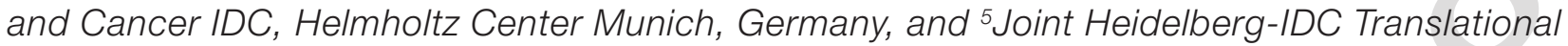
Diabetes Program, Dept. Inner Medicine I, Heidelberg University Hospital, Germany

${ }^{*}$ I.H.H. and T.F. contributed equally to this work

BioTechniques 61:137-148 (September 2016) doi 10.2144/000114452

Keywords: flow cytometry; fluorescence activated cell sorting (FACS); high-throughput screening (HTS); protein aggregation; yeast prion; amyloid aggregates; Saccharomyces cerevisiae

Supplementary material for this article is available at www.BioTechniques.com/article/114452.

Protein aggregation is both a hallmark of and a driving force for a number of diseases. It is therefore important to identify the nature of these aggregates and the mechanism(s) by which the cell counteracts their detrimental properties. Currently, the study of aggregation in vivo is performed primarily using fluorescently tagged versions of proteins and analyzing the aggregates by fluorescence microscopy. While this strategy is considered the gold standard, it has several limitations, particularly with respect to its suitability for high-throughput screening (HTS). Here, using a GFP fusion of the well-characterized yeast prion amyloid protein [PSI+], we demonstrate that flow cytometry, which utilizes the same physical principles as fluorescence microscopy, can be used to determine the aggregate load and pattern in live and fixed yeast cells. Furthermore, our approach can easily be applied to high-throughput analyses such as screenings with a yeast deletion library.

Misfolding of proteins and their accumulation into aggregates causes a diverse spectrum of age-associated fatal diseases such as neurodegenerative diseases as well as others such as vascular inflammation and atherosclerosis (1-4). It is currently not known why misfolded proteins and/or their accumulation into aggregates can become detrimental to the cell, but recent studies suggest that the sequestration of aggregates into specialized deposition sites is a key defensive strategy for protecting the cell from harmful interactions involving the misfolded proteins (5-8). Understanding the cellular strategies of aggregate handling is therefore key to developing therapeutic approaches for treating those diseases associated with misfolded proteins.

The mechanism(s) for the handling of aggregates have been extensively studied in the model organism Saccharomyces cerevisiae $(9,10)$. The separation and characterization of aggregated and non-aggregated protein species by traditional biochemical methods has proven useful, but these procedures are sometimes rather laborious and consequently often inapplicable for large screenings (11). The current gold standard for characterizing protein aggregates is fluorescence microscopy using fluorescently tagged substrates that are known to aggregate. This method, however, has some signif- icant limitations with respect to compatibility with high-throughput screening (HTS) $(12,13)$. For instance, the speed of image acquisition can be rate-limiting, especially at the higher magnification that is required for small model organisms such as yeast. When image acquisition is automated, the type of autofocus used can also greatly reduce the speed of image acquisition. Furthermore, using multiple fluorescence channels may be time-consuming, particularly when working with fluorochromes of different quantum yields. This time-cost aspect also often limits such screening to fixed cells. Automated image analysis has helped to reduce this burden, but it requires the generation of highly sophis-

\section{METHOD SUMMARY}

Our simple flow cytometry-based method allows rapid characterization of protein aggregation in living yeast cells using GFP fusions of model amyloid proteins. In addition to differentiating between the soluble or aggregated state of a protein, this method also allows for the characterization of the load and distribution of aggregates in individual cells within a large population and is easily applicable to high-throughput screening (HTS). 
ticated algorithms, which, if not used correctly, can be error-prone. The large amount of image data generated by automated microscopy also requires large memory storage capacities. Due to these limitations, it has been suggested that HTS using automated microscopy should be simplified when possible by reducing the number of fluorescence channels, limiting the number of images that are acquired per well, or using a low magnification (12). Following an initial screen, a more detailed analysis can then be performed on the subset of positive candidates and/or conditions identified by the screening.

Researchers have therefore started to investigate other methods to characterize aggregation. Since protein aggregation can be associated with toxicity, a readout related to diminished cell growth is particularly useful for HTS (14-16). However, experimental conditions that allow for detection of a correlation between aggregation, toxicity and altered cell growth are often hard to find and rather indirect. Recently, flow cytometry was successfully employed for aggregate characterization in mammalian cells (13). Characterization and quantification of differently sized aggregates from yeast is also possible using flow cytometry, but only ex vivo, once the cells have been lysed (17).

Flow cytometry measures optical and fluorescence characteristics of single cells in suspension as they pass through a beam of light. In this respect, flow cytometry is similar to microscopy, except that instead of producing an image of a cell, it automatically quantifies a set of parameters, which include a cell's relative size and reflective index, referred to as the forward scatter (FSC); granularity/internal complexity referred to as side scatter (SSC); and the relative fluorescence intensity of a fluorophore (18), which in yeast would be the fluorescently tagged protein. As yeast containing a fluorescently tagged protein pass through the light beam, photons are emitted, and the intensity of the voltage for that given fluorochrome increases. As the yeast completes its path through the light beam, it leaves behind a pulse of voltage over time (Supplementary Figure S1). This pulse is defined by its height $(H)$ and width (W), both of which are measured by the flow cytometer. A cell in which the fluorescence is evenly distributed will generate a broad pulse (low $\mathrm{H}$ and high W), whereas a cell with punctate fluorescence will generate a narrower, steeper pulse (high $\mathrm{H}$ and low W). The area under the pulse, referred to as the pulse area, is directly proportional to the intensity of the fluorescence for that event and can therefore be used as a measure of the expression and/or abundance of the fluorescently tagged protein. Of the three parameters that define the pulse, the $\mathrm{W}$ parameter can also be used to characterize the relative distribution of the fluorochrome of interest. Using this parameter, frequency analysis can be performed and then fitted by nonlinear regression to a Gaussian distribution. The resulting curve is defined by the mean of the $\mathrm{W}$ signal, the spread or standard deviation (SD) of the W signal, and the maximum percent frequency or amplitude. Of these parameters, it is the amplitude and SD that can be used to define the distribution of the fluorochrome signal within the cell. Such pulse-shape analysis cannot be readily performed by conventional fluorescence microscopy, which is limited to the single parameter of signal intensity. It has been demonstrated that flow cytometry and pulse-shape analysis can be used to monitor changes in the localization of GFP-tagged polyglutamine (polyQ)-expanded Huntingtin protein in mammalian cells (13).

Here, we investigate the viability of flow cytometry as a screening tool to characterize protein aggregation in yeast. As a model system for aggregation, the amyloidogenic yeast $\left[\mathrm{PS}^{+}\right]$prion was selected. The amino terminal PrD domain of the Sup35 protein is responsible for the prion properties of $\left[\mathrm{PSI}^{+}\right]$(19), and prions can be easily visualized using fusions with GFP (20-22). In the aggregated state, the prion can be present in different forms. This can range from a single high-intensity focus (23) representing an insoluble protein deposit (IPOD) (24), to multiple intense foci, distributed apparently at random within the cytoplasm (25). These different phenotypes depend on various experimental factors, such as the choice of the prion protein fusion construct or the level of expression used (26). Loss of the prion state and its concomitant aggregation can be observed as a result of the deletion and/ or inhibition of the molecular chaperone Hsp104 (27). These different aggregation states in otherwise isogenic yeast strains were initially used to establish whether analysis by flow cytometry could detect differences in the aggregation status of $\left[\mathrm{PSI}^{+}\right] / \mathrm{PrD}-\mathrm{GFP}$ in intact yeast cells.

\section{Materials and methods}

Plasmids, yeast strains, and standard growth conditions

The yeast strain Y8205 (28) was modified by deletion of the PrD domain in the endogenous SUP35 protein by a replacement of wild-type SUP35 with pSup35C using a pop-in/pop-out method (29,30), followed by an insertion of pRS306-GPD-PrD-GFP (23) at the Cyc1 terminator. This created a strain that became [psi], but stayed $\left[P / N^{+}\right]$because it continuously harbored the Rnq1 protein in the prion state. After several rounds of restreaking and selection for the prion phenotype (one single fluorescent focus) (23), a strain that propagated PrD-GFP in the mature prion form was obtained. Curing with $5 \mathrm{mM}$ guanidine hydrochloride (GndHCl) (Sigma-Aldrich, Munich, Germany) resulted in a [pin'] strain that harbored PrD-GFP in the soluble non-prion form (23). Proteins Htt25Q and Htt103Q were expressed from the cen plasmids p416-Gal-Htt25Q/103Q (31) in BY4741 (Euroscarf, Frankfurt, Germany). Gene deletions were introduced into the modified Y8205 strain by mating it to strains of the yeast deletion library (Invitrogen, Carlsbad, CA) following the systematic gene analysis (SGA) strategy (32).

Unless otherwise indicated, yeast cells were grown in standard YPD media (components purchased from BD Diagnostics, Sparks, MD) at $30^{\circ} \mathrm{C}$ to an $\mathrm{OD}_{600}$ of $\sim 0.5$ and then transferred to PBS (Sigma-Aldrich) for flow cytometry analysis and fluorescence microscopy.

\section{Yeast deletion library} screening using SGA

A screen of the first plate of the yeast deletion library was done according to SGA (32). Details are included in the Supplementary Materials.

\section{Fluorescence microscopy}

Fluorescence microscopy was performed to validate flow cytometry data using standard microscopy methods. Details are included in the Supplementary Materials.

\section{Flow cytometry}

Cells were analyzed at a low flow rate in a BD FACSCanto system equipped with a 488 nm laser (BD Biosciences, San Jose, CA). For each sample, 50,000 events were collected. Data for the GFP/FITC channel were collected as pulse height, area, and width parameters. 
A
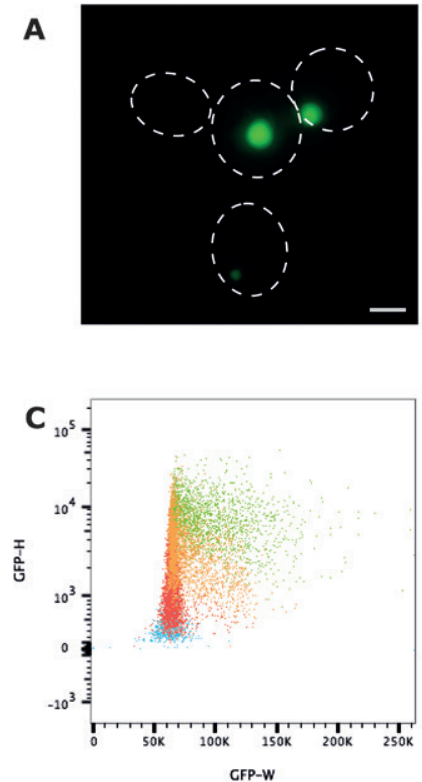

$\mathbf{E}$

\begin{tabular}{|c|c|c|c|c|c|c|}
\hline Color & $\begin{array}{c}\text { Subpopulation } \\
\text { description }\end{array}$ & \%Parent & $\begin{array}{c}\text { GFP } \\
\text { intensity }\end{array}$ & $\begin{array}{c}\text { IPOD size } \\
\text { (pixel } \\
\text { units*1000) }\end{array}$ & $\begin{array}{c}\text { Calcofluor } \\
\text { White } \\
\text { intensity }\end{array}$ & $\begin{array}{c}\text { Average } \\
\text { number of } \\
\text { bud scars }\end{array}$ \\
\hline Blue & Low GFP-A & 13.5 & 566 & 2,20 & 2015 & $0-1$ \\
\hline Red & Medium GFP-A & 41.7 & 1894 & 3,68 & 4192 & $1-4$ \\
\hline Orange & High GFP-A & 28 & 5179 & 8,70 & 9326 & $1-6$ \\
\hline Green & Very high GFP-A & 16.8 & 12575 & 32,38 & 21355 & $3-9$ \\
\hline
\end{tabular}

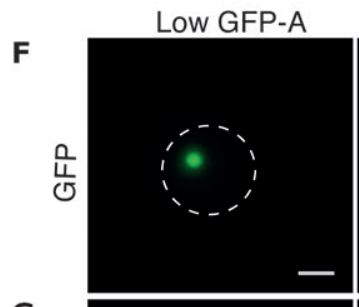

Medium GFP-A

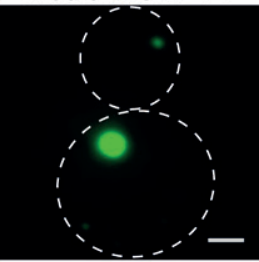

High GFP-A

Very high GFP-A

G
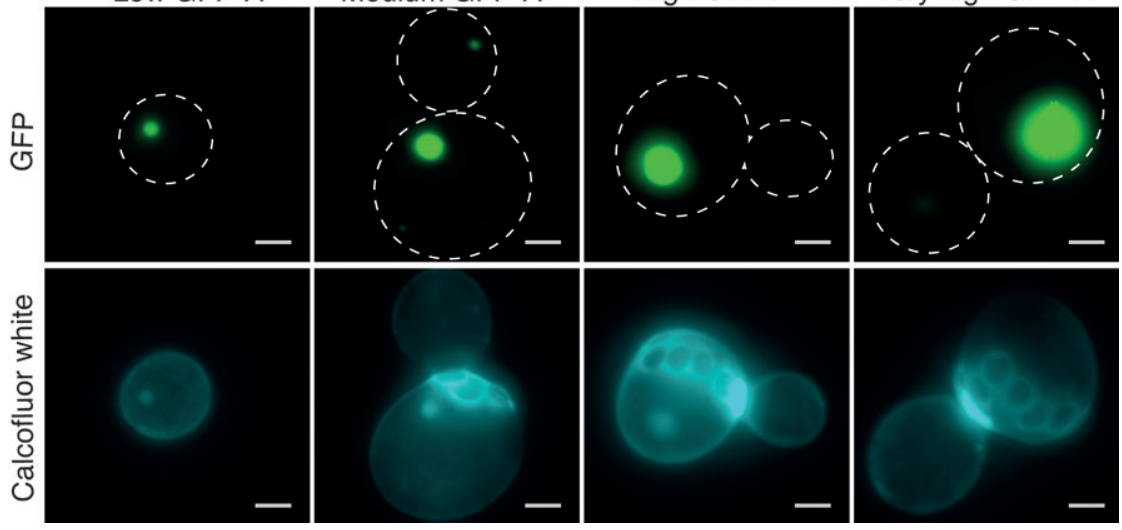

Figure 1. Characterization of a yeast strain harboring aggregates of the PrD-GFP prion by fluorescence microscopy, flow cytometry, and cell sorting. (A) Fluorescence micrograph of the yeast strain. (B) GFP-A versus forward scatter (FSC)-A scatter plot displaying gating according to size and distribution of GFP signal in the four subpopulations: Low GFP-A, Medium GFP-A, High GFP-A, and Very High GFP-A. (C) GFP-H (height) versus GFP-W (width) scatter plot of the GFP+ populations. (D) Scatter plot of GFP-A versus calcofluor white-A. (E) Table describing several parameters of the four subpopulations. (F) Fluorescence microscopy analyses of the four subpopulations-Low GFPA, Medium GFP-A, High GFP-A, and Very High GFP-A - that were collected during FACS sorting. (G) Fluorescence microscopy in the calcoflour white channel of the same subpopulations as in (F). Dashed lines represent the cell borders; scale bar: $2 \mu \mathrm{m}$. Each scatter plot represents 50,000 events.

For cell sorting and subsequent imaging by microscopy, cells were collected into a $5 \mathrm{~mL}$ polypropylene FACS tube by a FACSAria cell sorter equipped with a 488 $\mathrm{nm}$ and $405 \mathrm{~nm}$ (for the detection of calcoflour white) laser (BD Biosciences). These cells were then imaged by fluorescence microscopy.
For the high-throughput screening, cells were analyzed at a low flow rate in a BD FACSCanto, equipped with a $488 \mathrm{~nm}$ laser and a 96-well plate sample loader (BD Biosciences). In the screening application, 10,000 events/sample were collected

Flow cytometry data was analyzed using FlowJo version xV0.7 (Ashland OR).
For pulse-shape analysis, flow cytometry data in FCS format were exported into text format using FlowJo and analyzed in GraphPad Prism version 6.05 for Mactonish (GraphPad Software, San Diego, CA). Frequency distribution analysis of GFP-W signal was performed, tabulating the data and plotting it as relative frequency in percentages. Nonlinear regression was then performed, fitting data to a Gaussian curve, to determine signal amplitude, SD, and mean GFP-W signal.

A detailed step-by-step protocol is provided in the Supplementary Material.

\section{Results and discussion}

Detection of aggregate

load by flow cytometry

To establish the characteristics of the PrD-GFP aggregation model, cells harboring the protein in the mature prion state were analyzed by fluorescence microscopy and flow cytometry. Analysis by flow cytometry revealed that the culture was considerably more heterogeneous, particularly with respect to the amount of GFP fluorescence (GFP-A), than anticipated by microscopy where PrD-GFP aggregates were localized uniformly to one single aggregate corresponding to the IPOD (Figure 1A) (23). Based upon the GFP-A versus FSC/size scatter plots, the $\mathrm{GFP}^{+}$population was divided into four sub-populations: GFPvery high, GFPhigh, GFPmedium, and GFPlow (Figure 1B). This would suggest a different aggregate load of PrD-GFP within individual cells. This heterogeneity was confirmed by the scatter plots of the height (GFP-H) versus width (GFP-W) of the GFP signal (Figure 1C), which showed that GFP-H varied considerably, from 0 to $10^{4}$ arbitrary units. However, the range for GFP-W was narrower, indicating a similar cellular distribution of PrD-GFP. To confirm differences in PrD-GFP loads between individual cells, fluorescence activated cell sorting (FACS) was used to collect different GFP sub-populations (Figure 1E). Microscopy analysis showed that GFPvery high cells had a bigger, more intense focal aggregate (Figure 1F, right panel) as compared with GFPhigh, GFPmedium (Figure 1F, middle two panels), or GFPlow (Figure $1 F$, left panel) cells.

One feature that would contribute to unequal amounts of PrD-GFP aggregates in individual cells is an asymmetrical distribution of the PrD-GFP aggregates during cell division. This process ensures that 
Single aggregate

A

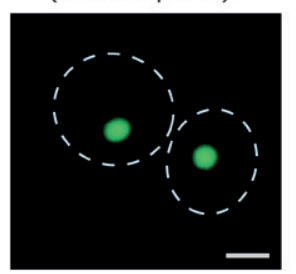

B

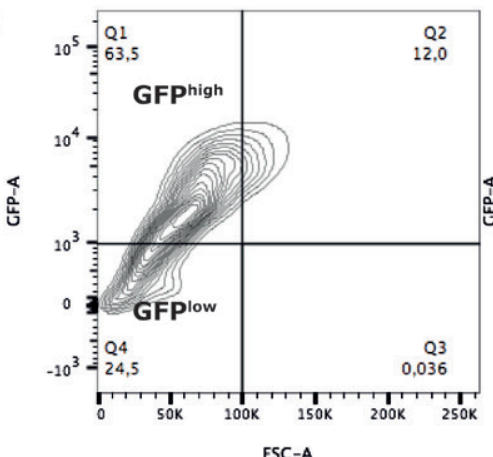

C
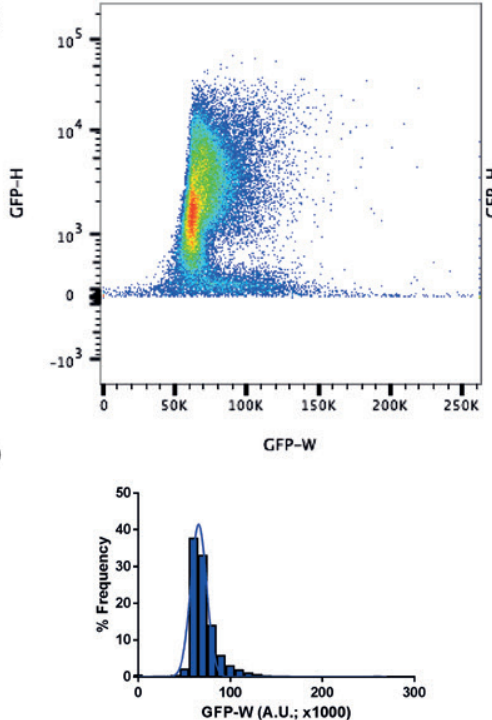

E

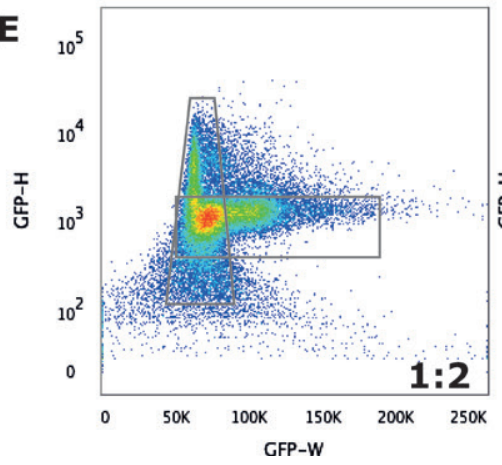

Soluble/Diffuse

(Prion cured)
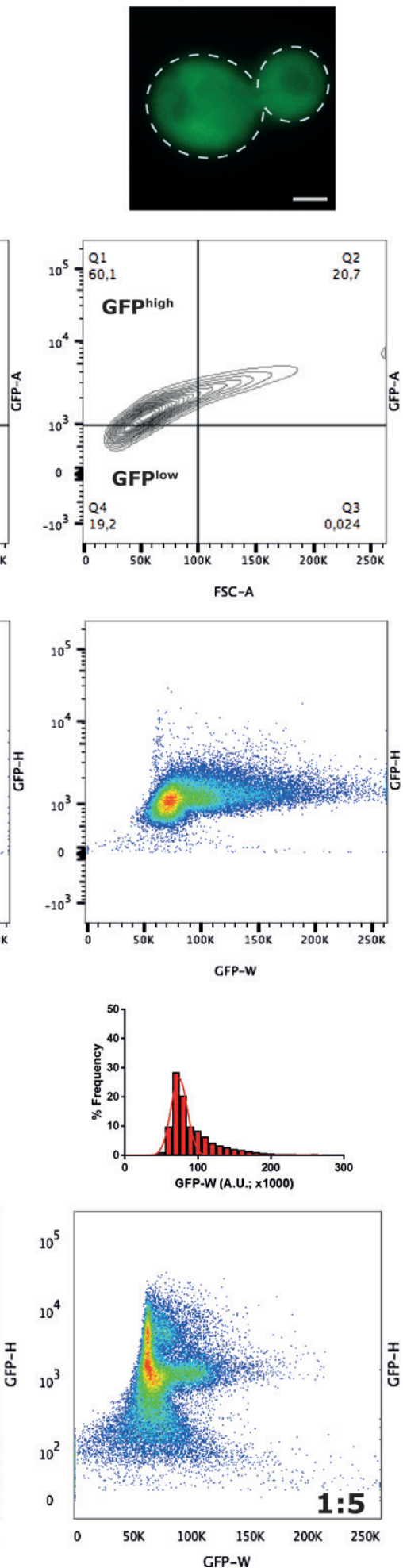

Soluble/Diffuse

(Ahsp104)
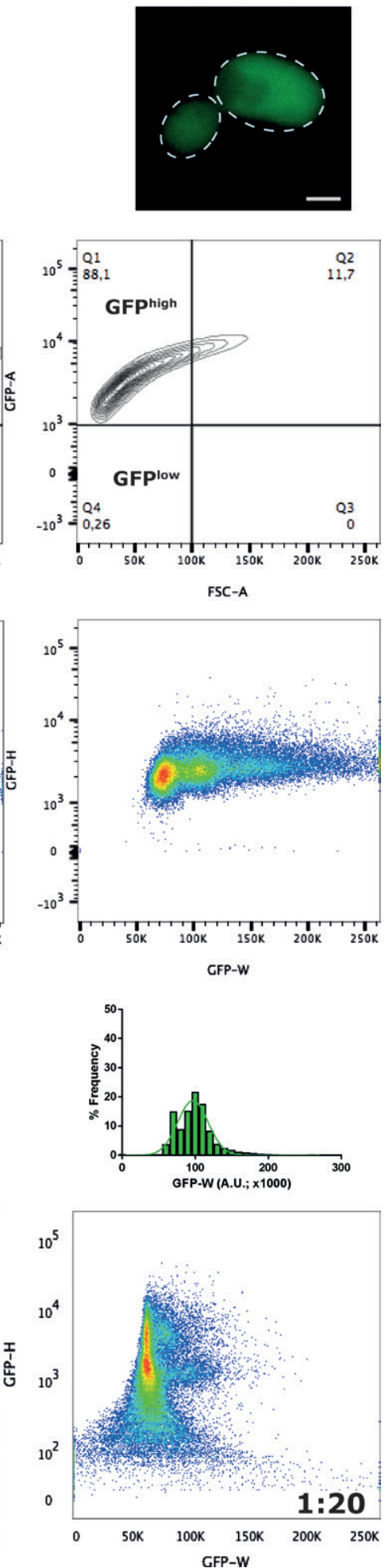

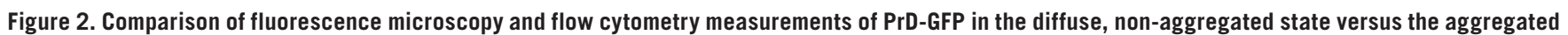

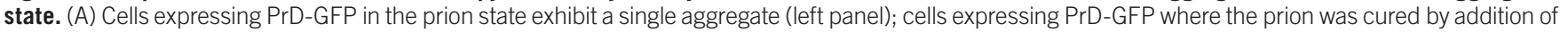

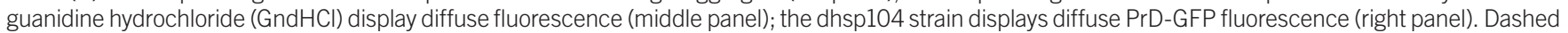

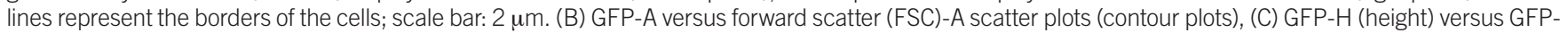

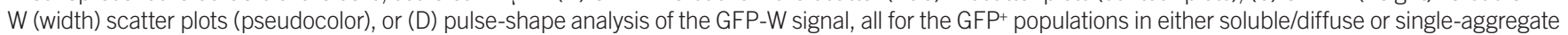

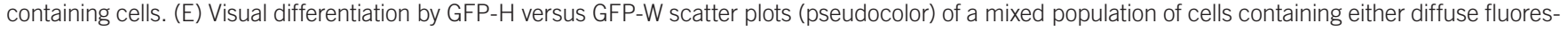

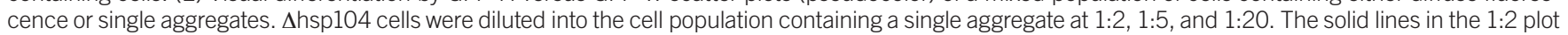

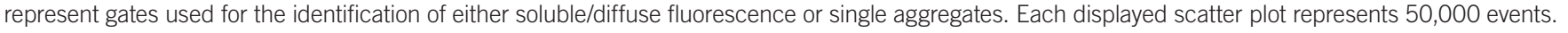


the burden of aggregated proteins is not passed on to the daughter cell but rather is retained by the mother (23,33-35). As a consequence, older cells would have a higher aggregate load as compared with freshly budded ones. The replicative age of yeast cells can be estimated by determining the number of bud scars after staining with calcoflour white. Utilizing a flow cytometer equipped with the appropriate lasers and emission filters, the intensities of PrD-GFP and calcoflour white could be measured simultaneously. It was found that cells from populations with higher GFP intensities also had higher calcofluor white signals as compared with the cells from GFP populations with lower GFP intensities (Figure 1, $D$ and E). Microscopy analysis (Figure 1G) confirmed this correlation between GFP and calcoflour white intensities measured by flow cytometry and the number of bud scars and size of the PrD-GFP IPODs analyzed by microscopy (Figure 1E), further supporting the idea of asymmetric aggregate distribution during cell division. The GFPvery high population (17\% of the cells) was consistently considerably smaller than the GFPhigh and GFPmedium populations (Figure 1E). This is in agreement with the observation that the number of old cells within a logarithmically growing yeast culture is rather small.

Differences in the aggregation

status of PrD-GFP can be

detected by flow cytometry

To establish whether flow cytometry could also be used to distinguish between PrD-GFP in the soluble and aggregated state, the mature prion state with a focal aggregate (Figure 2A, left panel) was compared with a culture where the prion state was removed by curing via inhibition of Hsp104 with low concentrations of $\mathrm{GndHCl}(23,36)$, resulting in a diffuse fluorescence signal (Figure 2A, middle panel). Additionally, we included $\Delta$ hsp104 cells where PrD-GFP was also diffusely distributed in the cytoplasm (Figure 2A, right panel). Analysis by flow cytometry showed that each culture tested had a unique scatter plot with respect to GFP-A versus FSC (Figure 2B). In comparison to the scatter plot of cells with a single aggregate (Figure 2B, left panel), the soluble/diffuse aggregation phenotype consisted of a single GFP population (Figure 2B, middle and right panels) with a much narrower range of GFP intensity (GFP-A). There was almost no difference between the $\mathrm{GnDHCl}$-cured and the $\Delta$ hsp104 cells, suggesting that the differences observed between the cells with either diffuse fluorescence or single aggregates were due to different distributions of the PrD-GFP protein and were not artifacts of $\mathrm{GndHCl}$-curing or differences in the $\Delta$ hsp104 knockout background. We also consistently observed no difference between the three strains with respect to growth rate (Supplementary Figure S2) that could account for the differences observed by flow cytometry. The differences between the two phenotypes became even clearer in the GFP-H versus GFP-W scatter plots (Figure 2C). The cells bearing a single aggregate (Figure 2C, left panel) had a much broader spread of GFP-H values but a narrower range of GFP-W values as compared with diffuse cells (Figure 2C, middle and right panels). This was consistent with the more variable amounts of PrD-GFP localized to a single focus as compared with PrD-GFP in the diffuse state (Figure 2A) because soluble PrD-GFP is not subject to retention in the mother cells, and its distribution is more

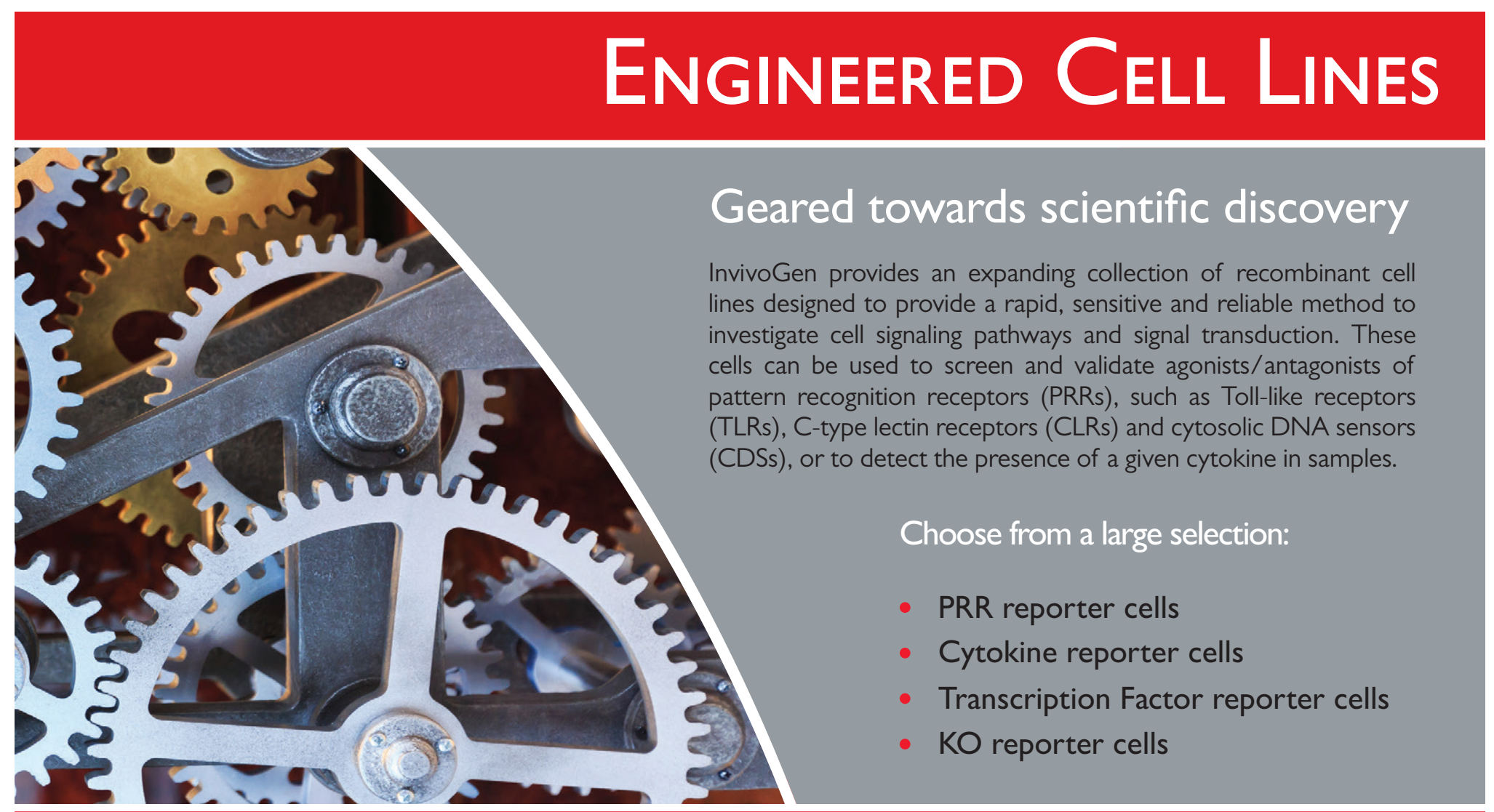



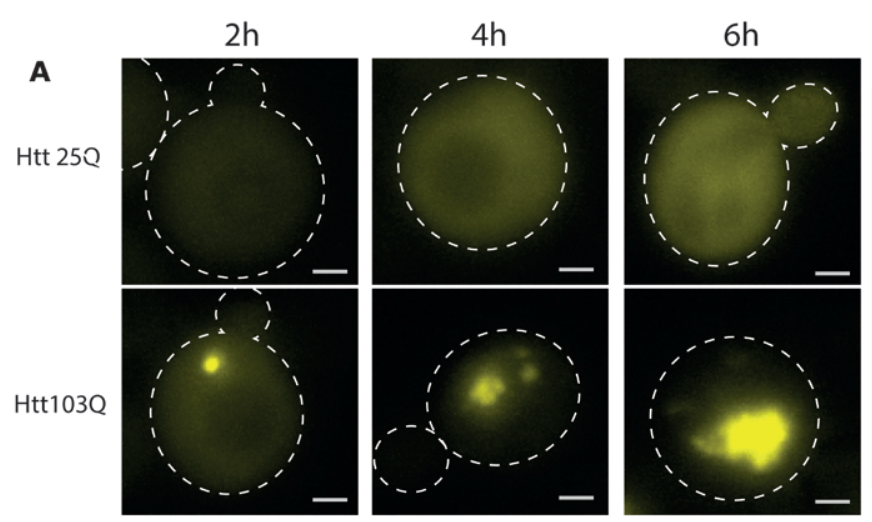

\begin{tabular}{|c|c|c|}
\hline $\begin{array}{c}\text { Average } \\
\text { GFP } \\
\text { intensity }\end{array}$ & Htt25Q & Htt103Q \\
\hline 2h & 413 & 547 \\
\hline $4 h$ & 1229 & 1315 \\
\hline 6h & 2223 & 2258 \\
\hline $\begin{array}{c}\text { \% of cells } \\
\text { with } \\
\text { aggregates }\end{array}$ & Htt25Q & Htt103Q \\
\hline $\begin{array}{c}\text { 2h } \\
\text { 4h }\end{array}$ & 0 & 29 \\
\hline $6 h$ & 0 & 97 \\
\hline & & 100 \\
\hline
\end{tabular}
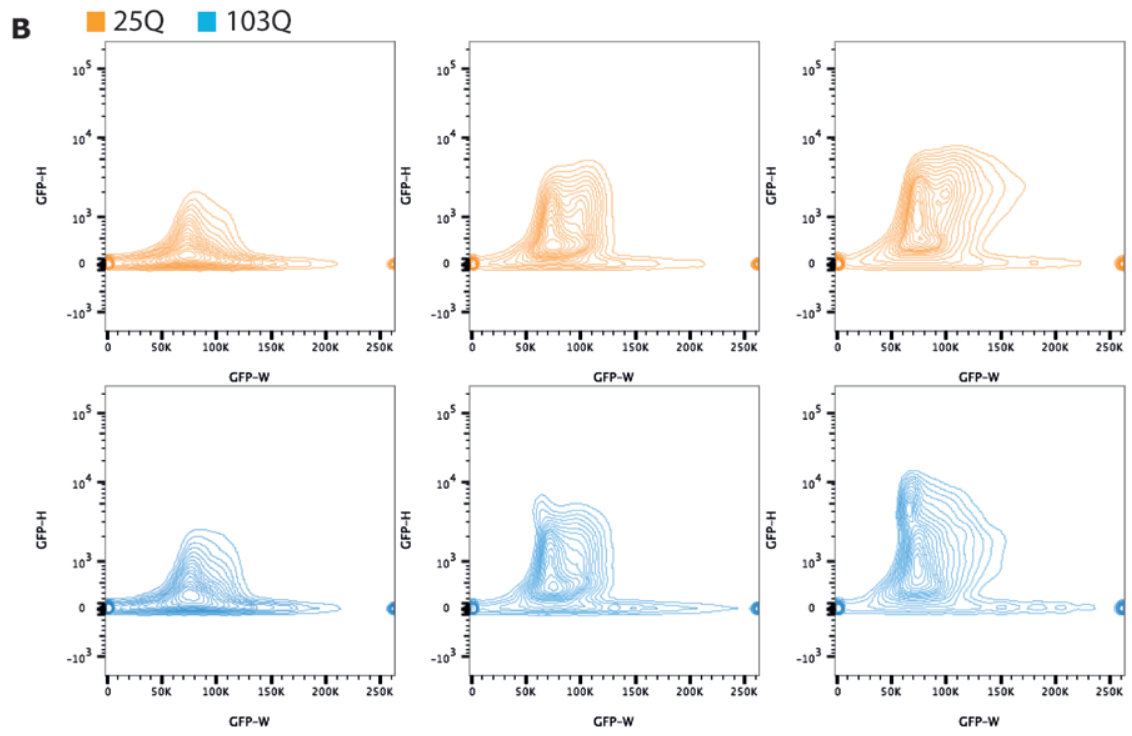

C
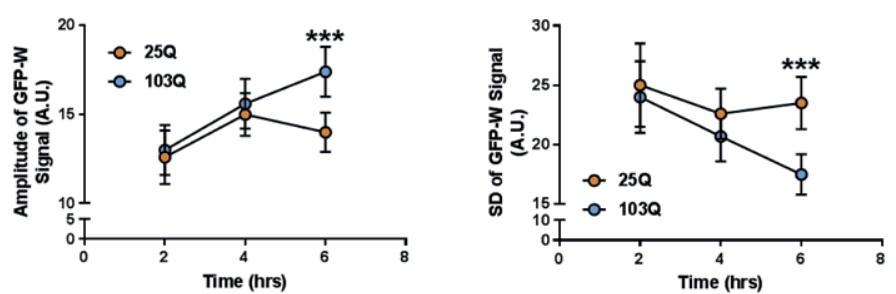

Figure 3. Characterization by flow cytometry of yeast strains expressing Huntingtin exon 1 constructs Htt25Q and Htt103. (A) Fluorescence microscopy images of Htt25Q (upper panels) and Htt103Q (lower panels) expressing strains after 2, 4, and $6 \mathrm{~h}$ of galactose induction. The dashed lines represent the borders of the cells, and the scale bar represents $2 \mu \mathrm{m}$. Average GFP intensities of $\mathrm{Htt} 25 \mathrm{Q}$ and $\mathrm{Htt} 103 \mathrm{Q}$ strains at different induction time points as well as percentages of cells with aggregates are given in the table to the right. (B) GFP-H (height) versus GFP-W (width) scatter plots of cells expression Htt25Q (upper panels) or Htt103Q (lower panels). Each scatter plot represents 50,000 events (cells). (C) Pulse-shape analysis of the GFP-W signal of cells expressing Htt25Q (orange) or Htt103Q (blue) showing variation of the amplitude (left panel) and SD (right panel) of the GFP-W signal at different time points of induction.

dependent on the size and shape of each individual cell.

To better describe the differences observed, pulse-shape analysis was performed. For the two aggregate phenotypes tested, the parameters of the pulseshape analysis are given in Supplementary Table S1. Comparison of the soluble/diffuse phenotype to the single foci aggregate showed that the latter had a significant increase in the amplitude of the GFP-W signal, as well as a significant decrease in the SD, giving rise to a higher and narrower pulse signal (Figure 2D), consistent with the previously described scatter plots and microscopy analysis.

To further demonstrate the specificity and sensitivity of the detection of these two aggregation states by flow cytometry, the soluble/diffuse cells were diluted into a population of cell containing a single aggregate. Analysis of the GFP-H versus
GFP-W scatter plots clearly showed the presence of two distinct populations, even at the highest dilution of 1:20, which corresponded to the appropriate aggregation phenotype of the mixed cells (Figure 2E).

Different aggregation substrates can be characterized by flow cytometry To demonstrate whether analysis by flow cytometry could be applied to other aggregating protein substrates, the first exon of the Huntington's disease protein, Huntingtin $(\mathrm{Htt})$, either with a short polyQ stretch (Htt25Q) or a long polyQ extension (Htt103Q), which is the aggregating form, were studied. For this experiment, we induced expression of the proteins by galactose administration for different periods of time to yield different expression levels and then measured the average GFP fluorescence intensity (Figure 3A). Preliminary analysis of the GFP-H versus GFP-W scatter plots showed less pronounced differences between $\mathrm{Htt} 25 \mathrm{Q}$ and $\mathrm{Htt} 103 \mathrm{Q}$ at early times of induction of the constructs (Figure 3B, left panel), but these differences became more obvious with prolonged expression times and concomitantly higher protein levels (Figure 3B, right panel). Pulseshape analysis (Figure 3 C) reflected these changes; for the Htt103Q aggregating construct, during prolonged induction the amplitude increased and the SD decreased, with the subsequent GFP peak becoming steeper and narrower. Similar changes were not observed for Htt25Q (Figure 3C). These changes are consistent with those previously described between the diffuse signal and single-aggregate cells containing the prion substrate, suggesting that $\mathrm{Htt} 25 \mathrm{Q}$ displays the diffuse fluorescence phenotype while Htt103Q's is more aggregated. These phenotypes were subsequently confirmed by fluorescence microscopy (Figure 3A), demonstrating that the different aggregating substrates can be characterized by flow cytometry.

\section{Limitations of protein aggregation} characterization by flow cytometry The aggregation status of the Htt protein highlights one of the major limitations of this flow cytometry method, in that when the expression levels of the protein of interest are very low (short induction times), the differences measured by flow cytometry are less obvious as compared with longer expression times, where more protein accumulates (Figure 3B). Furthermore, 
at early time points of induction, some of the Htt103Q protein was still soluble (Figure 3A, left panel). Thus, when the protein of interest only aggregates partially, the differences in the plots are also less obvious. This demonstrates that, despite the flexibility of the flow cytometer, it may not necessarily have the same degree of sensitivity as a fluorescence microscope. However, such limitations can be overcome by ensuring that the cytometer is fully calibrated with respect to the laser and optical setup (see protocol in Supplementary Material). Furthermore, a preliminary analysis of a sample of the culture with and without the fluorescently tagged protein of interest should be run prior to the implementation of the method. This is to determine whether the signal of the fluorescently tagged protein of interest is higher than the background/auto-fluorescence of the culture and can therefore be easily differentiated from non-fluorescent cells. If this is not possible, then experimental adjustments would be required to ensure either a higher level of expression, more complete aggregation, or a brighter fluorochrome in order to facilitate the detection of differences by flow cytometry.

High-throughput analyses of aggregate load and distribution by flow cytometry To establish whether HTS by flow cytometry could be used as part of genome-wide screening of the yeast deletion library, a query strain harboring a PrD-GFP fusion in the prion state (23) was prepared and mated with the first plate of the yeast deletion library, following the SGA procedure (32), so as to introduce the gene deletions into the strain background that propagated PrD-GFP in the prion state. Cultures of the resulting haploid cells were then screened by flow cytometry. The majority of the strains analyzed displayed GFP-H versus GFP-W plots that were indistinguishable from the single-aggregate phenotype of the wild-type strain (data not shown). However, eight gene deletions had scatter plots that were markedly distinct from the wild-type strain (Figure 4 and Supplementary Figure S3, middle panels). The most obvious difference was observed in the $\Delta$ hsp104 strain present in the first plate of the library, which had previously been used to characterize the diffuse fluorescence phenotype (Figure 2). Furthermore, three gene deletions,
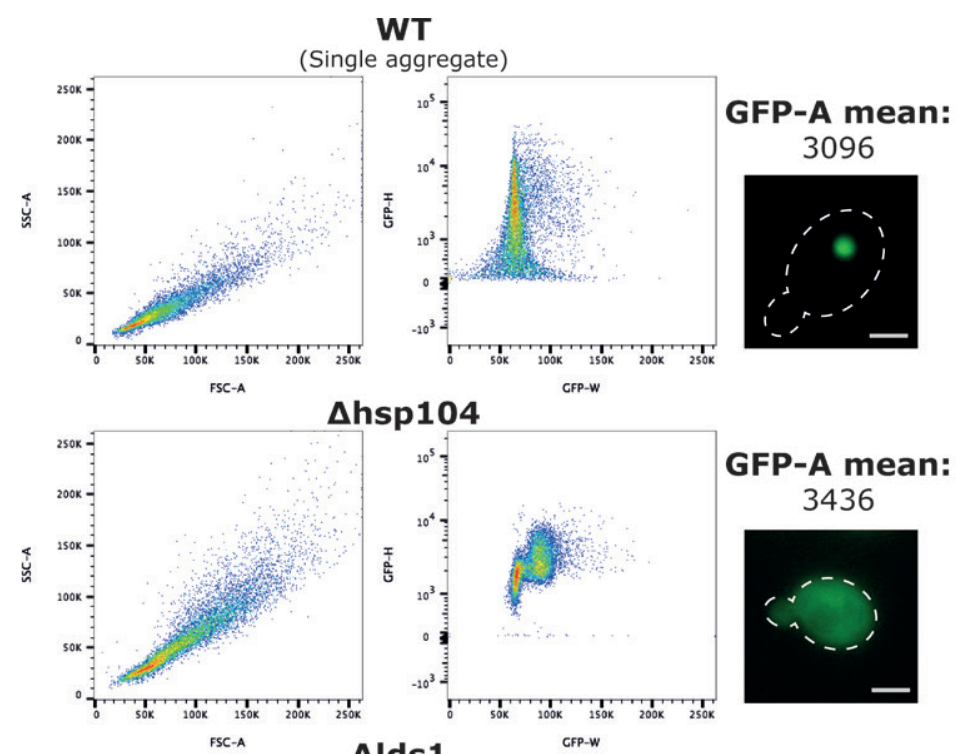

GFP-A mean: 3436

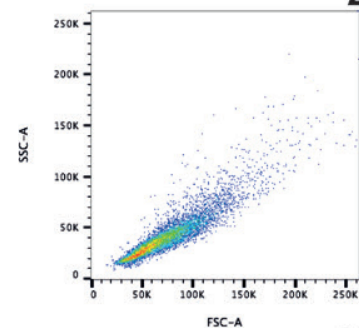

$\Delta$ Ids1
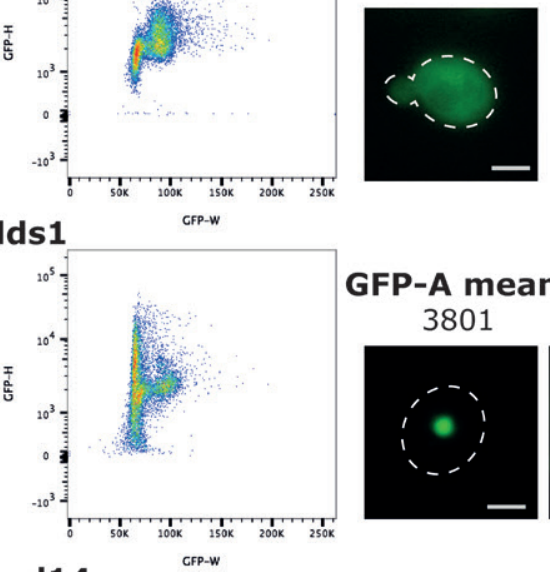

GFP-A mean: 3801

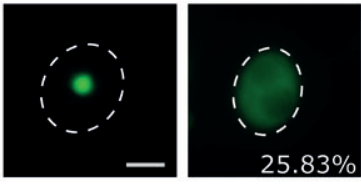

$\Delta$ bud14
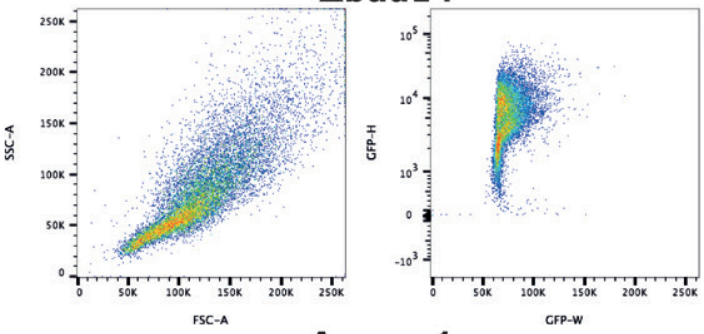

GFP-A mean: 8311
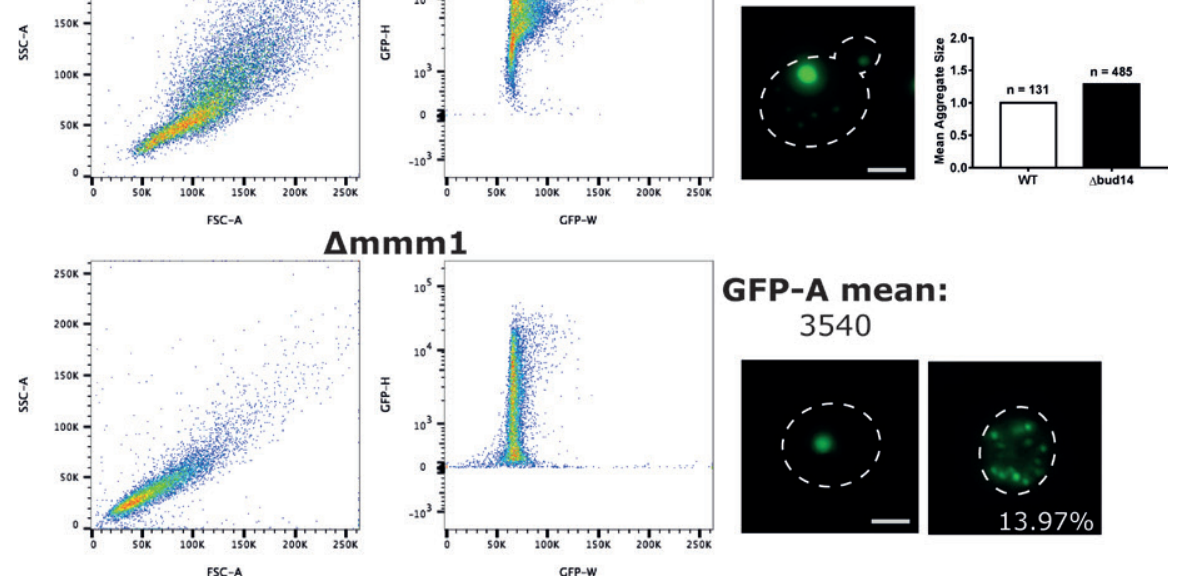

$\Delta \mathrm{mmm1}$

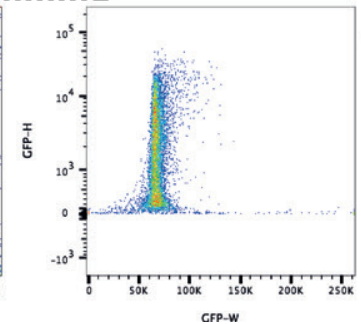

GFP-A mean: 3540

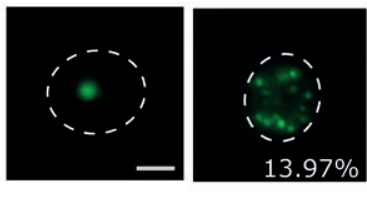

Figure 4. Hits from a screen for aggregate load and distribution of PrD-GFP in the first plate of the yeast deletion library. Pseudocolor scatter plots of the side scatter (SSC)-A versus forward scatter (FSC)-A (left panels) or GFP-H (height) versus GFP-W (width) of the GFP+ population (middle panels) and the corresponding fluorescence microscopy images (right panels) of different yeast deletion strains as indicated, generated through systematic gene analysis (SGA) of the first plate of the yeast deletion library and a yeast strain constitutively expressing PrD-GFP in the prion state (1 single aggregate/cell). Average GFP fluorescence intensity per cell/event in arbitrary units (GFP-A mean), measured by flow cytometry, is given. Percentages in the fluorescence images correspond to the frequency of the phenotype shown. For the $\Delta$ bud14 strain in comparison to the wild-type, the average "single aggregate" size was determined by single particle analysis using Image J software; " $\mathrm{n}$ " is the number of cells included in the analysis. All strains were analyzed directly after SGA as non-fixed cells. The dashed lines represent the borders of the cells, and the scale bar represents $2 \mu \mathrm{m}$. Each scatter plot represents 10,000 events.

$\Delta$ lds1 (Figure 4), $\Delta$ pex22, and $\Delta y a l 004 w$ (Supplementary Figure S3) had scatter plots that resembled the ones from the mixing experiments in which $\Delta$ hsp104 cells were mixed into a population of wild-type cells (Figure 2E). The YALO04W ORF is classified as dubious, but overlaps with the SSA1 ORF coding for an Hsp70-type 
chaperone involved in prion inheritance (37-39). Microscopy analysis confirmed that there was a considerable percentage of cells with diffuse fluorescence $(7 \%-26 \%)$ also present in the cultures that were predominantly cells with a single focal PrD-GFP aggregate (Figure 4 and Supplementary Figure S3, right panels). Although such a phenotype may make sense for cells with impaired SSA1 protein function, it was unexpected in this case because the $\mathrm{N}$-terminal part of the protein is involved in prion maintenance $(40,41)$, but the deletion of the dubious YALO04W ORF removes the C-terminal part of the protein. Thus, the biological significance has to be further determined for all three of the identified genes. Nonetheless, FACS analysis was able to show these differences among the deletion strains in a sensitive and reliable manner.

A second group of gene deletions within a subpopulation of cells with much higher GFP-H and GFP-W signals as compared with a wild-type culture was also identified (Figure 4 and Supplementary Figure S3, middle panels). These were BUD14 (Figure 4), PMT2, and KIN3 (Supplementary Figure S3), in which the mean intensity of the GFP signal per cell (right panels) was clearly higher. Furthermore, the relative size and morphology of a subpopulation of cells in these mutants, which can be seen in the SSC-A versus FSC-A plot (Figure 4 and Supplementary Figure S3, left panels), was larger. Bigger cells would contain more PrD-GFP, which could explain the higher GFP intensity per cell. Fluorescence microscopy followed by singleparticle analysis of the single-aggregate cells confirmed that the cells had larger aggregates (Figure 4 and Supplementary Figure S3, right panels). Although in this case it is probably more related to cell morphology rather than prion biology, such a phenotype would have been harder to identify in a large-scale fluorescence microscopy approach. One further interesting hit was the MMM1 gene, in which a clear subpopulation of cells with lower GFP-H values was observed that was not present in the wild-type strain (Figure 4, middle panel). Fluorescence microscopy revealed in this case that $\sim 14 \%$ of the cells contained many tiny aggregates rather than one single focal aggregate, which would correspond to this subpopulation.
Considering the full range of parameters measured by flow cytometry, it is possible not only to reveal differences in aggregation behavior but also to explain some of the observed differences. However, a more detailed analysis by fluorescence microscopy of the most promising single hits of a screen is still required to reveal their possible biological significance.

Our HTS confirmed that flow cytometry can easily be employed for genome-wide screening applications. This should be true not only for genomic libraries, but also for small molecule/compound libraries that are usually much more extensive and require less expensive and time-intensive solutions.

\section{Author contributions}

I.H.H. performed all experiments, was responsible for data interpretation, and wrote the manuscript with T.F. and J.T. T.F. assessed in all experiments, helped with data interpretation, and wrote the manuscript with I.H.H. and J.T. V.E. provided assistance with setting up and running the flow cytometer and helped with data interpretation. S.H. contributed to the conceptional framework of the study. P.P.N. organized the scientific surroundings for the project and contributed to the writing of the manuscript. J.T. supervised the project and wrote the manuscript together with I.H.H and T.F.

\section{Acknowledgments}

This study was supported by the Deutsche Forschungsgemeinschaft to Jens Tyedmers (TY93/1-1), and the SFB1118 (to P.P.N and T.F), the Dietmar Hopp Foundation (DHS) and the German Center for Diabetes Research (DZD). We would like to thank Matthias Meurer and Michael Knop for establishing the settings for sample application for FACS analysis in a 96-well format and their excellent help with the screening procedure. We thank the FACS Facility of the ZMBH, headed by Monika Langlotz, for excellent technical assistance. We also thank Axel Mogk for critical comments on the manuscript.

\section{Competing interests}

The authors declare no competing interests.

\section{References}

1. Hipp, M.S., S.H. Park, and F.U. Hartl. 2014. Proteostasis impairment in protein-misfolding and -aggregation diseases. Trends Cell Biol. 24:506514.

2. Kikis, E.A., T. Gidalevitz, and R.I. Morimoto. 2010. Protein homeostasis in models of aging and age-related conformational disease. Adv. Exp. Med. Biol. 694:138-159.

3. Knowles, T.P., M. Vendruscolo, and C.M. Dobson. 2014. The amyloid state and its association with protein misfolding diseases. Nat. Rev. Mol. Cell Biol. 15:384-396.

4. Buchberger, A., B. Bukau, and T. Sommer. 2010. Protein quality control in the cytosol and the endoplasmic reticulum: brothers in arms. Mol. Cell 40:238-252

5. Arrasate, M., S. Mitra, E.S. Schweitzer, M.R Segal, and S. Finkbeiner. 2004. Inclusion body formation reduces levels of mutant huntingtin and the risk of neuronal death. Nature 431:805-810.

6. Tanaka, M., Y.M. Kim, G. Lee, E. Junn, T. Iwatsubo, and M.M. Mouradian. 2004. Aggresomes formed by alpha-synuclein and synphilin-1 are cytoprotective. J. Biol. Chem. 279:4625-4631.

7. Wolfe, K.J., H.Y. Ren, P. Trepte, and D.M. Cyr. 2013. The Hsp70/90 cochaperone, Sti1, suppresses proteotoxicity by regulating spatial quality control of amyloid-like proteins. Mol. Biol. Cell 24:3588-3602.

8. Olzscha, H., S.M. Schermann, A.C. Woerner, S. Pinkert, M.H. Hecht, G.G. Tartaglia, M. Vendruscolo, M. Hayer-Hartl, et al. 2011. Amyloid-like aggregates sequester numerous metastable proteins with essential cellular functions. Cell 144:67-78.

9. Summers, D.W. and D.M. Cyr. 2011. Use of yeast as a system to study amyloid toxicity. Methods 53:226-231.

10. Miller, S.B., A. Mogk, and B. Bukau. 2015. Spatially organized aggregation of misfolded proteins as cellular stress defense strategy. J. Mol. Biol. 427:1564-1574.

11. Alberti, S., R. Halfmann, and S. Lindquist. 2010. Biochemical, cell biological, and genetic assays to analyze amyloid and prion aggregation in yeast. Methods Enzymol. 470:709-734.

12. Cohen, Y. and M. Schuldiner. 2011. Advanced methods for high-throughput microscopy screening of genetically modified yeast libraries. Methods Mol. Biol. 781:127-159.

13. Ramdzan, Y.M., S. Polling, C.P. Chia, I.H. Ng, A.R. Ormsby, N.P. Croft, A.W. Purcell, M.A. Bogoyevitch, et al. 2012. Tracking protein aggregation and mislocalization in cells with flow cytometry. Nat. Methods 9:467-470.

14. Cooper, A.A., A.D. Gitler, A. Cashikar, C.M. Haynes, K.J. Hill, B. Bhullar, K. Liu, K. Xu, et al. 2006. Alpha-synuclein blocks ER-Golgi traffic and Rab1 rescues neuron loss in Parkinson's models. Science 313:324-328.

15. Tyedmers, J., M.L. Madariaga, and S. Lindquist. 2008. Prion switching in response to environmental stress. PLoS Biol. 6:e294.

16. Giorgini, F., P. Guidetti, Q. Nguyen, S.C. Bennett, and P.J. Muchowski. 2005. A genomic screen in yeast implicates kynurenine 3-monooxygenase as a therapeutic target for Huntington disease. Nat. Genet. 37:526-531.

17. Shiber, A., W. Breuer, and T. Ravid. 2014. Flow cytometric quantification and characterization of 
intracellular protein aggregates in yeast. Prion 8:276-284.

18. Ormerod, M.G. 2000. Flow Cytometry: A Practical Approach, Third Edition. Oxford University Press, Oxford, U.K.

19. Tuite, M.F. 2000. Yeast prions and their prionforming domain. Cell 100:289-292.

20. Borchsenius, A.S., R.D. Wegrzyn, G.P. Newnam, S.G. Inge-Vechtomov, and Y.O. Chernoff. 2001. Yeast prion protein derivative defective in aggregate shearing and production of new 'seeds'. EMBO J. 20:6683-6691.

21. Patino, M.M., J.J. Liu, J.R. Glover, and S. Lindquist. 1996. Support for the prion hypothesis for inheritance of a phenotypic trait in yeast. Science 273:622-626

22. Zhou, P., I.L. Derkatch, and S.W. Liebman. 2001. The relationship between visible intracellular aggregates that appear after overexpression of Sup35 and the yeast prion-like elements [PSI(+)] and $\mathrm{PIN}(+)$. Mol. Microbiol. 39:37-46.

23. Tyedmers, J., S. Treusch, J. Dong, J.M. McCaffery, B. Bevis, and S. Lindquist. 2010. Prion induction involves an ancient system for the sequestration of aggregated proteins and heritable changes in prion fragmentation. Proc. Natl. Acad. Sci. USA 107:8633-8638

24. Kaganovich, D., R. Kopito, and J. Frydman. 2008. Misfolded proteins partition between two distinct quality control compartments. Nature 454:1088-1095.

25. Satpute-Krishnan, P. and T.R. Serio. 2005. Prion protein remodelling confers an immediate phenotypic switch. Nature 437:262-265.

26. Tyedmers, J. 2012. Patterns of [PSI (+) ] aggregation allow insights into cellular organization of yeast prion aggregates. Prion 6:191-200.
27. Chernoff, Y.O., S.L. Lindquist, B. Ono, S.G. IngeVechtomov, and S.W. Liebman. 1995. Role of the chaperone protein Hsp104 in propagation of the yeast prion-like factor [psi+]. Science 268:880-884

28. Tong, A.H. and C. Boone. 2006. Synthetic genetic array analysis in Saccharomyces cerevisiae. Methods Mol. Biol. 313:171-192.

29. Liu, J.J. and S. Lindquist. 1999. Oligopeptiderepeat expansions modulate 'protein-only' inheritance in yeast. Nature 400:573-576.

30. True, H.L., I. Berlin, and S.L. Lindquist. 2004. Epigenetic regulation of translation reveals hidden genetic variation to produce complex traits. Nature 431:184-187.

31. Duennwald, M.L., S. Jagadish, F. Giorgini, P.J. Muchowski, and S. Lindquist. 2006. A network of protein interactions determines polyglutamine toxicity. Proc. Natl. Acad. Sci. USA 103:1105111056

32. Tong, A.H., M. Evangelista, A.B. Parsons, H. Xu, G.D. Bader, N. Page, M. Robinson, S. Raghibizadeh, et al. 2001. Systematic genetic analysis with ordered arrays of yeast deletion mutants. Science 294:2364-2368.

33. Aguilaniu, H., L. Gustafsson, M. Rigoulet, and T. Nystrom. 2003. Asymmetric inheritance of oxidatively damaged proteins during cytokinesis. Science 299:1751-1753

34. Cox, B., F. Ness, and M. Tuite. 2003. Analysis of the generation and segregation of propagons: entities that propagate the $[\mathrm{PSI}+]$ prion in yeast. Genetics 165:23-33.

35. Liu, B., L. Larsson, A. Caballero, X. Hao, D. Oling, J. Grantham, and T. Nystrom. 2010. The polarisome is required for segregation and retrograde transport of protein aggregates. Cell 140:257-267.
36. Ferreira, P.C., F. Ness, S.R. Edwards, B.S. Cox, and M.F. Tuite. 2001. The elimination of the yeast $[\mathrm{PSI}+]$ prion by guanidine hydrochloride is the result of Hsp104 inactivation. Mol Microbiol. 40:1357-1369.

37. Rikhvanov, E.G., N.V. Romanova, and Y.O. Chernoff. 2007. Chaperone effects on prion and nonprion aggregates. Prion 1:217-222.

38. Shorter, J. and S. Lindquist. 2008. Hsp104, $\mathrm{Hsp} 70$ and $\mathrm{Hsp} 40$ interplay regulates formation, growth and elimination of Sup35 prions. EMBO J. 27:2712-2724.

39. Winkler, J., J. Tyedmers, B. Bukau, and A. Mogk. 2012. Hsp70 targets Hsp100 chaperones to substrates for protein disaggregation and prion fragmentation. J. Cell Biol. 198:387-404.

40. Sharma, D. and D.C. Masison. 2008. Functionally redundant isoforms of a yeast Hsp70 chaperone subfamily have different antiprion effects. Genetics 179:1301-1311.

41. Sharma, D. and D.C. Masison. 2011. Single methyl group determines prion propagation and protein degradation activities of yeast heat shock protein (Hsp)-70 chaperones Ssa1p and Ssa2p. Proc. Natl. Acad. Sci. USA 108:1366513670.

Received 10 March 2016; accepted 7 July 2016.

Address correspondence to Jens Tyedmers, Department of Medicine I and Clinical Chemistry, Im Neuenheimer Feld 410, University of Heidelberg, 69120 Heidelberg, Germany. E-mail: Jens.Tyedmers@med.uni-heidelberg.de

To purchase reprints of this article, contact: biotechniques@fosterprinting.com

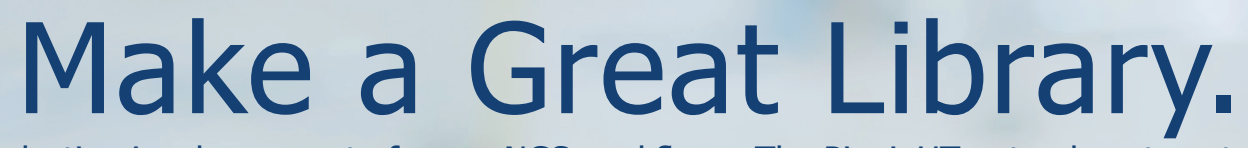

DNA size selection is a key aspect of many NGS workflows. The PippinHT not only automates this step, but improves the quality of results. For short-read sequencing or long-range genomics, make your library great.

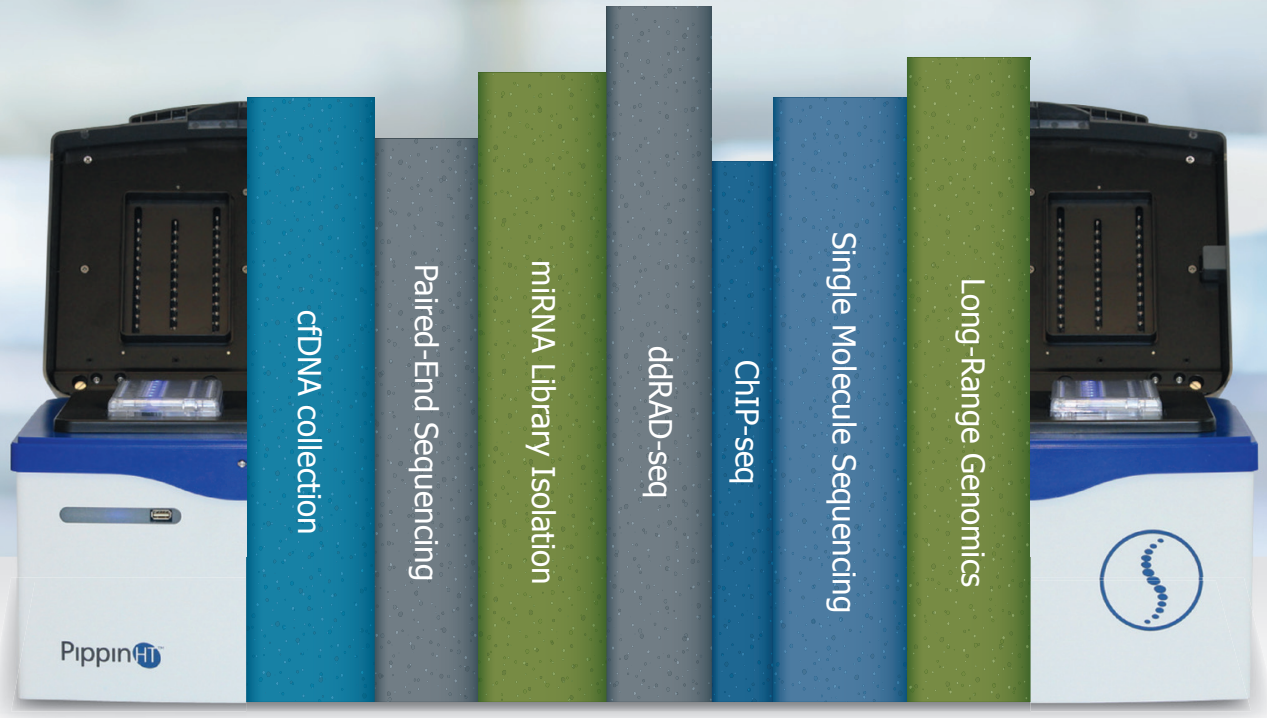

\section{PippinHT DNA Size Selection System}

- Precise and Reproducible

- Up to 24 Samples per Run
(1) sage science www.sagescience.com @SageSci 